\title{
Wilson's disease: the problem of delayed diagnosis
}

J M Walshe, $M$ Yealland

\begin{abstract}
To discover the earliest symptoms and signs of neurological Wilson's disease we analysed the case histories of 136 patients who were seen between 1955-87: patients with hepatic or presymptomatic Wilson's disease were excluded from this series. Thirty one patients (23\%) gave a history of an episode of liver damage. The onset of symptoms ranged from nine to 40 years with a median of 16.2 years. The correct diagnosis was made at presentation in only 43 patients. The mean delay before diagnosis was 12.8 months for the others. The earliest symptoms were dysarthria or difficulty with the hands, or often both. There was often an associated change in personality or deteriorating performance at school. The four common clinical pictures were Parkinsonian (61 cases), "pseudosclerotic"' (33 cases), dystonic ( 21 cases) and choreic (15 cases): six cases were unclassified. Parkinsonian symptoms were equally common in children (under 17 years) and adults, a "pseudosclerotic" picture was much more common in adults but dystonic and choreic symptoms were seen more often in children. Experience suggests that no two patients are ever the same, even in a sibship.
\end{abstract}

\section{(F Neurol Neurosurg Psychiatry 1992;55:692-696)}

More than thirty years of investigation and management of patients with Wilson's disease has shown that there is frequently an unacceptable delay in establishing the correct diagnosis. This is greatly to the disadvantage of the patient not only by prolonging unnecessarily the duration of the illness but also by affecting adversely the prospects for recovery. If too much damage is allowed to occur in the brain before the initiation of "decoppering" therapy then full restoration of function is difficult to achieve despite the remarkable improvements which have been observed in CT scan appearances. ${ }^{1}$

The mean delay in diagnosis for patients presenting with neurological symptoms in this series has been in excess of one year. The onset of hepatic Wilson's disease, its evolution and response to treatment has been described elsewhere. ${ }^{2}$ It might be argued that Wilson's disease is so rare that failure to recognise it soon after onset is not surprising. However, it is now established that the prevelance in the general population is 30 per million ${ }^{3-5}$ and approximately half of these will present with neurological symptoms. ${ }^{6}$ Thus in the United Kingdom, with a birth rate of between 600000 and 700000 , there will be on average 10 new patients presenting annually to all the neurological clinics in the British Isles.

Little attention has been paid in the literature to the very earliest neurological symptoms and signs of this disease except for brief mention in Scheinberg and Sternlieb's monograph. ${ }^{4}$ While we are largely in agreement with their observations it is our intention to describe in greater detail this important stage in the evolution of the illness.

In many cases it has been difficult to identify the very earliest symptoms. Most patients will have been seen by a number of doctors, often of different disciplines (general medical, psychiatric and neurological) and have had their case histories taken on numerous occasions before referral. Thus their recollection of how the illness started will have faded with the passage of time and will also have been influenced by direct questioning dictated by the preconception of the examining physician. Also, in many cases the later development of more dramatic symptoms and signs will have coloured the patient's view of the disease.

We believe that the analysis of so many case histories, all taken by one of us, over a period in excess of 30 years, has enabled us to establish the onset, evolution and varying clinical pictures of this disease. There appears to be only one thing which all patients have in common, apart from Kayser Fleischer corneal pigment, namely that they are all different. It is therefore unwise to rely on seeing "the typical picture of Wilson's disease" before making the diagnosis.

\section{Material and methods}

We selected for study 136 patients $(F=70$, $M=66$ ) presenting with neurological signs from some 250 cases of Wilson's disease seen between 1955-87, the majority at Addenbrooke's Hospital, Cambridge; patients seen subsequently were not included. One hundred and fourteen cases have been omitted because the diagnosis was made before the onset of neurological symptoms. Thirty one (23\%) gave a history of an earlier episode of liver damage the significance of which was not appreciated at the time. The clinical picture of hepatic Wilson's disease, its diagnosis and management of the presymptomatic stage have been described elsewhere. ${ }^{6-8}$ Eighty five patients came from the United Kingdom and 
51 from abroad; mostly the Mediterranean countries and the Near East.

All patients showed the classical biochemical lesion of Wilson's disease, that is a low serum copper, a low caeruloplasmin and a high serum "free copper" together with an increased urinary output of the metal. They also all had Kayser Fleischer corneal pigment, though not necessarily complete rings, ${ }^{9}$ when first seen by us.

All patients were examined by one of us (JMW) immediately after referral and the majority were examined by both of us separately. We relied upon the recollections of the patients and the letters of referring physicians for information about the earliest stages of the illness. A minority of patients only were seen soon after the onset of symptoms.

Wilson's disease has a recessive mode of inheritance so that it is probable that families will be seen in which more than one sibling is affected. If the diagnosis of Wilson's disease has already been confirmed in one sibling there should be no problem in making the correct diagnosis if a second sibling develops similar symptoms. In our series 16 families had more than one affected sibling and in one kinship first cousins developed neurological Wilson's disease. In eight sibships screening studies led to the diagnosis of presymptomatic Wilson's disease in a younger sibling. In three other families a second sibling had early symptoms of neurological involvement. In three families the diagnosis of Wilson's disease in the propositus was not followed up and a second sibling developed symptoms at a later date. In one family the diagnosis in the elder sibling was only made after a second sibling developed symptoms.

In another family the establishment of the diagnosis of Wilson's disease in an elder sibling with neurological symptoms led to the finding that her younger sister, who was being treated for chronic hepatitis, also had the same disease. Interestingly, the 43 patients in whom the diagnosis of Wilson's disease was made at the first time of investigation, nine had affected siblings, whereas in the remaining 93 cases only three siblings had Wilson's disease.

The diagnoses made when the patients first consulted a doctor fall into four groups: 1) an organic disorder other than Wilson's disease $(35) ; 2)$ a psychiatric illness $(32) ; 3$ ) a group in which no diagnosis was made or else the patient declared fit (26); 4) Wilson's disease (43). Incorrect organic diagnoses were as diverse as flat feet, myxoedema, myasthenia gravis, encephalitis, multiple sclerosis and Parkinson's disease. Patients presented to paediatric, general medical, neurological and psychiatric clinics. Psychiatric diagnoses were schizophrenia, depression, anxiety state and hysteria. The type of problems patients suffer in the diagnostic process are illustrated by two brief case histories.

Case 1 had an excellent academic record until, at the age of 17 years, she developed "an acute anxiety state" which was attributed to the break-up of her parents' marriage. Following treatment with antidepressants she devel- oped Parkinsonian symptoms considered secondary to phenothiazines. During the next two years she suffered from increasing tremor of the arms and slurring of speech. She was referred to a consultant neurologist who considered her symptoms to be hysterical. As she became increasingly disabled she took an overdose of phenothiazines which was followed by exacerbation of her tremor. She was then referred to a psychiatrist who agreed with the diagnosis of hysteria. Later she was seen by a second neurologist who could find no evidence of organic disease. Six months later a third neurologist investigated her and, at that time, an ophthalmologist suggested that she might have Kayser Fleischer rings but this was ignored and again she was said to have no organic disease. Finally her speech defect became so severe that she was rendered mute. At that stage the partner in her father's general practice suggested the diagnosis of Wilson's disease and she was referred to us. We found that she had dense, broad, complete Kayser Fleischer rings, blepharospasm, saccadic eye movements, anarthria, severe action and resting tremor of the arms and truncal tremor. She eventually made an excellent recovery on penicillamine.

Case 2 a civil engineer noticed at the age of 30 years a change in his handwriting and in his speech which became quavering and rapid. This was followed by tremor of both arms. A diagnosis of multiple sclerosis was made and he was treated with ACTH. The following year he developed nodding tremor of the head and his work was seriously impeded. A consultant neurologist could find no abnormality in his speech but confirmed the tremor of the head and arms, the gait was normal. A cursory examination of the eyes did not reveal the presence of Kayser Fleischer rings. A CT brain scan showed dilatation of the ventricles, more marked on the left, and atrophic changes in the cerebral and in the cerebellar cortex. The serum caeruloplasmin was just below the lower normal limit for the laboratory. The diagnosis of Wilson's disease was discarded in favour of familial tremor. He was seen regularly for follow up and during this time there was a change in his personality, he took to drink and to hitting his wife who left him. This led to no review of the diagnosis or his management. After this he attended a neurological clinic in the United States where he was noted to have head tremor, action tremor of the arms and slurred speech. He was also found to have broad, dense complete Kayser Fleischer rings. He improved on treatment with penicillamine but developed immune complex nephritis. He was referred to us for treatment at this stage. He still had mild head and arm tremor and very dense corneal rings. He has subsequently made an excellent recovery on trientine.

The age of onset ranged from nine to 40 years with a median figure of 16 years, the median age for the onset of hepatic symptoms, in those patients who had shown them was 11 years (fig).

We have subdivided the patients into two groups, 65 juveniles (16 years of age and 


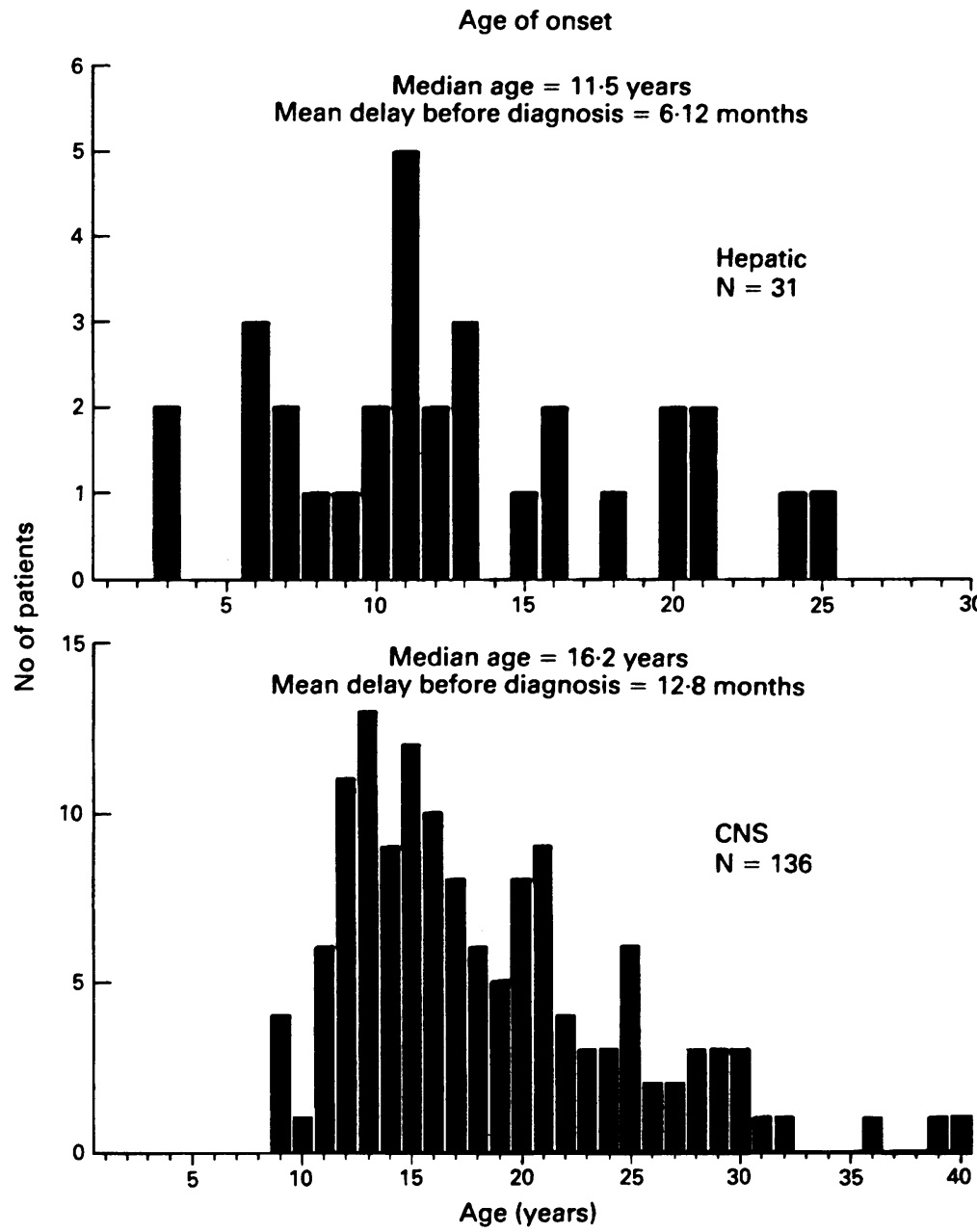

Figure This shows the age of onset of neurological symptoms in all 136 patients. $A$ total of 31 patients had an earlier episode of liver disease.

under) and 71 adults (17 years and over). The eight major complaints of personality change, speech defect, drooling, difficulty in swallowing, hand tremor, other difficulties with the hands, abnormal gait and a deterioration in the quality of school work, were largely similar in both groups though dysarthria and drooling were rather more common in the juveniles (table 1). Deterioration in school work could result from intellectual impairment, physical problems or both. The combination of a speech defect and drooling was found in $86 \%$ of juveniles (56) and $72 \%$ of adults (51); despite this fact difficulty in swallowing was not common in the early stages of the disease. In both children and adults dysarthria and tremor or clumsiness of the hands were the predominant early symptoms. Indeed one or both of these nearly always heralded the onset of the

Table 1 Number (percentage) of patients with different initial symptoms

\begin{tabular}{lll}
\hline Number of patients & $\begin{array}{l}\text { fuveniles } \\
65\end{array}$ & $\begin{array}{l}\text { Adults } \\
71\end{array}$ \\
\hline Personality change & $21(32)$ & $23(32)$ \\
Speech defect & $63(41)$ & $42(30)$ \\
Drooling & $31(20)$ & $22(16)$ \\
Dysphagia & $14(9)$ & $7(5)$ \\
Hand tremor & $48(31)$ & $55(39)$ \\
Hand clumsy & $32(21)$ & $20(14)$ \\
Abnormal gait & $34(22)$ & $18(13)$ \\
Fall in work at school & $40(26)$ & \\
\hline
\end{tabular}

disease. The speech defect could be of greatly varying severity. In a small number of patients a change in personality was the very earliest abnormality and showed itself as facile laughter, inappropriate and occasionally aggressive behaviour, labile mood, lack of insight and disinhibition.

The tremor was of varying severity and clumsiness of the hands was usually described as difficulty in writing. Problems in walking were not recorded as a first symptom in any patient though it often rapidly followed tremor of the hands. Despite the fact that the neurological lesion is biochemically induced and therefore affects the brain uniformly, limb symptoms and signs were always asymmetrical in the early stages of the disease. Muscle cramps and spasms were rare but did occur and presumably were responsible for the spontaneous dislocation of the jaw complained of, as a first symptom, by two patients; a third patient had such severe abnormal jaw movements that she needed dental extractions to protect her cheeks from damage by her teeth. Nine patients gave a history of epilepsy, either grand mal or focal fits, early in the illness; in one patient a single grand mal seizure was the only complaint when he was first seen, but examination revealed complete Kayser Fleischer rings and sun flower cataracts. Epilepsy was more common in juveniles (7) than in adults (2). A detailed account of epilepsy in Wilson's disease has already been presented. ${ }^{10}$ We observed no differences in symptoms between the sexes.

The signs, for both juveniles and adults, in the four diagnostic categories are shown in tables 1 and 2 . These will be described together as most differences seen in the pattern of signs in the 2 age groups are probably explained by the relatively small numbers involved. The one exception was that a Parkinsonian facies was noted three times more frequently in the juveniles than in the adults. In 10 cases no abnormal signs were recorded even though the patients may have complained of speech disturbances or tremor. Presumably this was because of the intermittent nature of the early movement disorders. Signs in the limbs were always asymmetrical with no preference for either side. As might be expected from the symptoms the commonest signs were dysar-

Table 2 Number (percentage) of juveniles and adults with different initial signs

\begin{tabular}{lrr}
\hline & Fuveniles & \multicolumn{2}{c}{ Adults } \\
& 65 & 71 \\
\hline Personality disorder & $14(21)$ & $13(18)$ \\
Dysarthria & $34(52)$ & $19(27)$ \\
Gait abnormal & $8(12)$ & $5(7)$ \\
Eye movement abnormal & $4(6)$ & $4(6)$ \\
Drooling & $18(28)$ & $11(15)$ \\
Parkinsonian facies & $15(23)$ & $5(7)$ \\
Open mouth & $10(15)$ & $0(0)$ \\
Bradykinesia & $6(9)$ & $3(4)$ \\
Tongue abnormal & $11(17)$ & $9(13)$ \\
UL tremor & $17(26)$ & $23(32)$ \\
UL dystonia & $14(21)$ & $13(18)$ \\
UL spontaneous movements & $8(12)$ & $0(0)$ \\
LL tremorN & $2(3)$ & $6(8)$ \\
LL dystoniaN & $12(18)$ & $0(0)$ \\
LL spontaneous movementsN & $11(1)$ & $1(1)$ \\
Liver disease & $19(29)$ & $8(11)$ \\
\hline
\end{tabular}

`UL \% upper limb. NLL \% lower limb. 
thria (53) and tremor of the hands (40 cases).

Although patients with dysarthria often had abnormal tongue movements these did not always occur together. Dysarthria varied from slight slurring of speech to complete incomprehensibility; it could involve disturbances of lip, tongue, palate, vocal cord and diaphragmatic movements. The incidence of abnormalities of speech was followed by that of Parkinsonian expression and by the change in personality. Disorders of eye movement, though not common, were seen; six patients were recorded as showing nystagmus while two gave a history of relatively short episodes of diplopia. The combination of an alert expression of the upper face with a rather vacuous open-mouthed lower face, so characteristic of the established disease, was recorded in only 10 juveniles and in no adults. Disorders of lower limb function were common at this stage; the gait was described as abnormal in eight children but in none of the adults.

The distribution of signs, no matter what the initial diagnosis, showed few variations among the patients. However, personality change was noted twice as often in patients who were labelled "psychiatric" as in the other groups, whether juvenile or adult. Kayser Fleischer rings were reported in 21 children and 13 adults in the correctly "diagnosed group" but in no others. In only one patient, diagnosed at this stage, were Kayser Fleischer rings sought with a slit lamp and not seen. When this patient was referred to us six months later the rings were dense and complete, despite six months chelation therapy. One patient in the "psychiatric" group was reported by an ophthalmologist as having Kayser Fleischer rings but this was ignored by the physician. Evidence of previous liver disease was most frequently seen in the "juvenile Wilson's disease" group.

In the patients not correctly diagnosed at the first consultation there was a mean delay of 13 months before it was established that they had Wilson's disease. Although the symptoms and signs of Wilson's disease are pleomorphic we found that it was possible to place all but six of the patients into four main clinical types: 1) Parkinsonian; 2) "pseudosclerotic"; 3) dystonic; 4) choreic. The Parkinsonian patients showed paucity of expression and movement. Although the term "pseudosclerosis"10 has largely been abandoned we have used it here because it is the best description of a type of the disease in which tremor closely resembles that seen in multiple sclerosis and which can be severe enough to be described as "wingbeating". The dystonic patients showed hypertonicity often associated with abnormal limb movements which could be grotesque. In the choreic group abnormal movements, which could be choreic or choreo-athetoid were often associated with dystonia. A Parkinsonian picture is the most common in both children and adults (table 3). The "pseudosclerotic" presentation occurs about three times more frequently in adults than in children whilst involuntary movements are seen six times more often in juveniles than in adults.
Table 3 Distribution of cases in four clinical types

\begin{tabular}{lrll}
\hline Number of patients & $\begin{array}{l}\text { fuveniles } \\
\text { Adults }\end{array}$ & \multicolumn{1}{l}{$\begin{array}{l}\text { Total } \\
71\end{array}$} & \multicolumn{1}{c}{136} \\
\hline Parkinsonian & $28(43)$ & $33(46)$ & $61(45)$ \\
Pseudosclerotic & $7(11)$ & $26(37)$ & $33(24)$ \\
Dystonic & $14(21)$ & $7(10)$ & $21(15)$ \\
Choreic & $13(20)$ & $2(3)$ & $15(11)$ \\
Intermediate & $3(5)$ & $3(4)$ & $6(4)$ \\
\hline
\end{tabular}

Percentage totals in brackets.

By the time the correct diagnosis was made many of the juveniles had moved into the adult group so comparison here is no longer valid. Predictably the physical signs had become more obvious and widespread with dysarthria and drooling remaining the most common. A striking feature in many patients was a peculiar and characteristic laugh on inspiration, (once heard never forgotten). The frequency of speech difficulties were closely followed by tremor, dystonia and spontaneous movements of the upper limbs. By this stage difficulty in walking and bradykinesia were much more common. Kayser Fleischer rings were noted in 135 patients and sun flower cataracts in three. In one patient, in whom the diagnosis was made retrospectively, the rings were not sought.

\section{Discussion}

Our findings in 136 patients with neurological Wilson's disease demonstrate that this diagnosis must seriously be considered in every adolescent who complains of a change in speech and difficulty in the use of the hands. A change in personality makes the diagnosis of Wilson's disease that much more probable. The correct diagnosis at the time of presentation was made in only 43 patients or less than one third; children fared slightly better than adults. For the others there was a mean delay of 13 months before the diagnosis was made, 35 were diagnosed as having an organic disease, 32 a psychiatric disorder and in 26 no diagnosis was made or the patients were declared fit. There was no striking difference in symptoms between these four groups of patients. Whenever the diagnosis is suspected, Kayser Fleischer rings should be sought by an experienced ophthalmologist, using a slit lamp. The rings or crescents can easily be missed by an inexperienced observer particularly if the iris is brown. Similarly laboratory errors in the estimation of copper and caeruloplasmin can delay the correct diagnosis; experience in the laboratory is as important as in the clinic. All adolescents and young adults with changes in personality and disturbances of movement, particularly involving speech and use of the hands, should be considered as possible examples of Wilson's disease if the sensory nervous system is not involved. As a screening procedure we recommend that the serum copper and caeruloplasmin should be determined and the eyes examined for Kayser Fleischer rings by slit lamp.

It is interesting to compare our findings with the case reports in Wilson's original publica- 
tion. ${ }^{11} \mathrm{He}$ observed, personally, four patients whose neurological symptoms showed a slightly older age of onset than our median age of 16 years, the youngest was 17 and the oldest 25 years. Two gave a clear history of an earlier episode of liver damage. In three patients the onset appeared to have been Parkinsonian while the fourth showed rigidity and "clonic tremor". One had an earlier and apparently self limiting psychotic episode.

Denny Brown ${ }^{12}$ divided his patients into two clinical types, the first he called "pseudosclerotic" and these were characterised by dysarthria and a flapping tremor of the "wingbeating" type, the patients were all over 19 years of age and the illness very chronic. The second group showed the progressive lenticular degeneration of Wilson and were all between the ages of seven and 15 years. The first symptom was "almost always related to dystonia". Some patients showed a Parkinsonian facies and fine tremor of the fingers. This latter group of patients responded very badly to treatment with dimercaprol. In this publication Denny Brown did not comment on the very earliest symptoms except to mention that there was often a tendency to inattentiveness at school, an observation in keeping with our own findings.

The delay in making a correct diagnosis is another feature of Wilson's disease which has received little attention in the literature. With increasing awareness of the disease over the past 30 years the diagnosis is being made more often than previously but the delay recorded in this series of 13 months is unacceptably long. The source of referral of the patients made surprisingly little difference to this figure, 13.1 months (range six to 72 months) for 85 patients from the United Kingdom and $12 \cdot 3$ months (range two to 60 months) for 51 patients from abroad. It is impossible to know how many patients have died undiagnosed. By drawing attention to the earliest symptoms, we hope that this article will increase diagnostic awareness and reduce delays so that patients can start their treatment earlier in the course of the illness and thus have a better prospect of achieving full functional recovery.

Finally, we have included an extract from a letter by a patient describing the start of her illness. She presented to one of us (JMW) late in 1989 and is not included in this series.

"The copper must have been building up all the time in my brain, but no symptoms started to show until I was sixteen years old. I was in my last year at college when I started to lose concentration and take dizzy spells when I was working in the kitchen. I managed to finish my course and get my qualifications.

I got a job in the hospital as a general catering assistant in the staff dining room. But the dizzy spells came back, only this time they were a lot worse as one time I fainted. I knew something was wrong so I told my mum and we mentioned it to the doctor. I was made redundant after 5 weeks just before Christmas on December 22nd 1988.

In January I noticed I was getting worse, started dribbling and also noticed when I walked, I was staggering about as though I was drunk. When I sat in a chair I gradually started to lean forward as if I was going to fall. When trying to ride my bike, I couldn't keep my balance. These symptoms went on for a couple of months with frequent visits to the doctor to see what was wrong with me.

I then noticed that my hands started shaking and I couldn't stop them. I started to lose my temper, swearing and throwing things, which was totally out of character for me. I felt I was being possessed by some evil force and had lost control of my actions. I felt like taking an overdose but I couldn't, not after saving a friend's life after she took an overdose and slashed her wrists. I just couldn't go through with it.

In April 1989, I was admitted to hospital by my doctor. I was put through all the tests, lumber puncture, cat scan, ECG, EEG, magnetic brain scan, but it wasn't until they looked in my eyes that they saw the copper rings and looked up their books to find the name. The professor came to the room to tell me and my mum and dad what the disease was and how it was treated. I was started on the tablets straight away. The doctors were not promising a quick recovery-it would take time, and I would have good days and bad days.

I am still going for check ups to see a specialist in London every so often, but I am getting better every day.

I owe my life to my mum for sticking by me and putting up with the abuse I gave her, and for pushing the doctors to help me get better.

I never thought I would see my 20 th birthday, it was very special to me."

1 Williams FJB, Walshe JM. Wilson's disease. An analysis of the cranial computerised tomography appearances found in 60 patients and the changes found in response to in 60 patients and the changes found in response to treatmen

2 Walshe JM. Wilson's disease presenting with symptoms of hepatic dysfunction: A clinical analysis of eighty-seven patients. Quart f Med 1989;70:253-63.

3 Bachmann H, Lossner J, Gruss B, Ruchholtz U. Die Epidemiologie der Wilsonehen Erkranjung in der DDR und die derzeitige Problematik einer populationgenetischen Bearbeitung. Psychiat Neurol Med Psychol (Leipzig) 1979;31:393-400.

4 Scheinberg IH, Sternlieb I. Wilson's disease, vol XXIII. Major problems in internal medicine. Philadelphia: W B Saunders, 1984

5 Walshe JM. Wilson's disease. BMF 1984;288:1689.

6 Walshe JM. Wilson's disease. Handbook of Clinical Neurology, vol 5(49). In: Vinken PJ, Bruym GW, Klawans HL, ogy, vol 5(49). In: Vinken PJ, Bruym GW, Klawans HL,

7 Walshe JM. Diagnosis and treatment of presymptomatic Wilson's disease. Lancet 1988;ii:435-7.

8 Sternlieb I, Scheinberg IH. The prevention of Wilson's disease in asymptomatic patients. N Engl f Med 1968; 278:352-9.

9 Cairns JE, Walshe JM. The Kayser Fleischer ring. Trans Ophthalm Soc UK 1970;90:187-90.

10 Denny TR, Berrios GE, Walshe JM. Wilson's disease and epilepsy. Brain 1988;III:1139-55.

11 Westphal C. Uber eine dem Gilde der Cerebrospinalen grauen Degenerationen ahnliche Erkrankung des centralen Nervensystems ohne anatomischen Befund nebst einigen Bemerkungen uber paradose Contraction. Arch Psychiat Nervenkr 1983;14:87-134.

12 Wilson SAK. Progressive lenticular degeneration: a familial nervous disease associated with cirrhosis of the liver. Brain 1912;34:295-509.

13 Denny Brown D. Hepatolenticular degeneration (Wilson's disease). Two different components. $N$ Engl f Med 1964; 270:1149-56. 